\title{
Data fusion for automated pain recognition
}

\author{
Steffen Walter, Sascha Gruss, Harald Traue \\ Medical Psychology, Department Psychosomatic Medicine \\ and Psychotherapy \\ University of Ulm \\ Ulm, Germany \\ steffen.walter@uni-ulm.de \\ Markus Kächele, Friedhelm Schwenker \\ Institute of Neural Information Processing \\ University of Ulm \\ Ulm, Germany \\ markus.kaechele@uni-ulm.de
}

\author{
Philipp Werner, Ayoub Al-Hamadi \\ Institute for Information Technology and \\ Communications \\ University of Magdeburg \\ Magdeburg, Germany \\ philipp.werner@uni-ovgu.de
Adriano Andrade, Gustavo Moreira
Faculty of Electrical Engineering
Federal University of Uberlândia
Uberlândia, Brazil \\ aoandrade@feelt.ufu.br
}

\begin{abstract}
Conservative methods of pain scales do not allow for objective and robust measurement, which are restricted to patients with "normal" communication abilities. If valid measurement of the pain is not possible, treating the pain may lead to cardiac stress in risk patients, under perfusion of the operating field, over- or under-usage of analgesics and other problems in acute or chronic pain conditions. Pervasive computing technologies via biopotential and behavioral parameters may represent a solution of robust pain recognition in clinical context and everyday life. In this work, multi-modal fusion of video and biopotential signals is used to recognize pain in a person-independent scenario. For this purpose, participants were recruited to subject to painful heat stimuli under controlled conditions. Subsequently, a multitude of features via biopotentials and behavior signals has been extracted and selected from the available modalities. Biopotential and video features were fused with an early and late fusion, we could show that the classification between baseline vs. tolerance threshold has an accuracy of $80 \%$ via late fusion. The data support the concept of automated and objected pain recognition of experimental pain. There are plans for a clinical project in which detection will occur postoperatively in humans.
\end{abstract}

Keywords-automated pain recognition; biopotentials; video; multimodal data; machine learning; data fusion

\section{INTRODUCTION}

Pain is a very personal sensation that is difficult to interpret without any communication from the patient. Consequently, a method for objective measurement of pain would be beneficial, particularly in cases where the patient is not able to describe the pain in clinical context and also in everyday life $[2,5,20]$ (e.g. somnolent patients, patients suffering from dementia). The recognition of pain captures information about the suffering of a person over the time and is relevant to the dosage of analgesics.

Research interest in automatic pain recognition has focused largely on recognition of facial expressions in painful situations for example using the UNBC-McMaster shoulder pain expression archive database $[7,10]$ and only recently the in-depth investigation of biopotentials has led to encouraging findings [14, 15] for automated pain recognition. Predictions based on both modalities combined as it is already very common in other sub-disciplines of machine learning is still an unexplored area and no works exist that leverage information fusion and multi expert systems. This work aims to investigate fusion of biopotentials and video data to improve recognition of pain intensity in person-independent scenarios.

\section{DATASET AND FEATURE EXTRACTION}

\section{A. Participants}

In these experiments the BioVid Heat Pain database [16] is analysed. It comprises 90 participants $((1) 18-35$ years $(n=30$ years; 15 men, 15 women), (2) 36-50 years $(\mathrm{n}=30$; 15 men, 15 women), and (3) 51-65 years ( $\mathrm{n}=30 ; 15$ men, 15 women). A total of 86 subjects were included in the final analysis because four subjects were excluded because of limited data quality with regard to the EMG. Only healthy subjects were recruited. Pre-existing neurological conditions, chronic pain, cardiovascular disease, regular use of pain medication, and use of pain medication immediately before the experiment were applied as exclusion criteria.

\section{B. Experimental Protocoll}

The experimental setup consisted of a thermode that was used for pain elicitation. The intensity was calibrated for each participant such that it divided the range between two reference levels (pain starts and pain is barely bearable) into 3 equally spaced intervals. Each of the 4 different stimulation (T1 vs. T2 vs. T3 vs. T4) strengths was applied 20 times to give rise to a total of 80 responses. During the experiments, high resolution videos (from 3 different cameras), sensor data of a Kinect, and a biopotential amplifier were recorded. The biopotential channels included electromyography (EMG) of zygomaticus, corrugator and trapezius muscles, skin conductance level (SCL) and an electrocardiogram (ECG). 
All of the features were extracted from a window of 5.5 seconds (Fig. 1).

The study was conducted in accordance with the ethical guidelines set out in the WMA Declaration of Helsinki (ethical committee approval was granted: 196/10-UBB/bal).

\section{Biopotential Feature Extraction}

Feature extraction of the biopotentials was performed after channel dependent preprocessing. The EMG and ECG channels were filtered using a Butterworth bandpass filter with the frequency ranges of $[20 ; 250] \mathrm{Hz}$ and $[0.1 ; 250] \mathrm{Hz}$, respectively. This step was necessary to reduce noise and minimize the effects of trends in the signals. For the EMG signal an additional noise reduction procedure based on Empirical Mode Decomposition was applied [1]. For the EMG and SCL channel, a number of features based on signal amplitude and frequency such as peak height, peak difference, mean absolute difference, Fourier coefficients, bandwidth were computed as well as additional features based on entropy (approximate and sample entropy [12]), stationarity [3] and statistical moments. In the ECG signal, first the QRS complexes were detected then based on the differences between consecutive heart beats (RR intervals), the mean difference, the root mean sum of squared differences (RMSSD) as well as the slope of the regression line computed on the RR intervals, were calculated.

\section{Video Feature}

From the video modality we extracted facial expression and head pose features. The head pose is estimated from depth maps by fitting a generic head model to the measured point cloud [17]. This yields 3 rotation angles and 3 position parameters per frame. For facial expression features, we automatically detect facial landmarks on the mouth, right eye and right eyebrow with IntraFace [19]. Several distances between these landmarks measure facial deformation.
These distances were measured in 3D [17] and include eye to brow distance, eye closure and mouth height, among others. Deepening of the nasolabial fold were measured through the mean gradient magnitude in the corresponding image region.

Over time, each of these facial expression and head pose parameters yields a signal. For each signal we applied a lowpass filter and calculated the first and second temporal derivative of the resulting signal.

Next, we extracted statistical parameters of the low-pass filtered signal and its derivatives, namely the mean, median, standard deviation, range, inter-quartile range, inter-decile range and median absolute deviation. The extracted statistical parameters were used as facial expression and head pose features for the following analysis. The overall feature extraction method is described in detail in $[17,18]$.

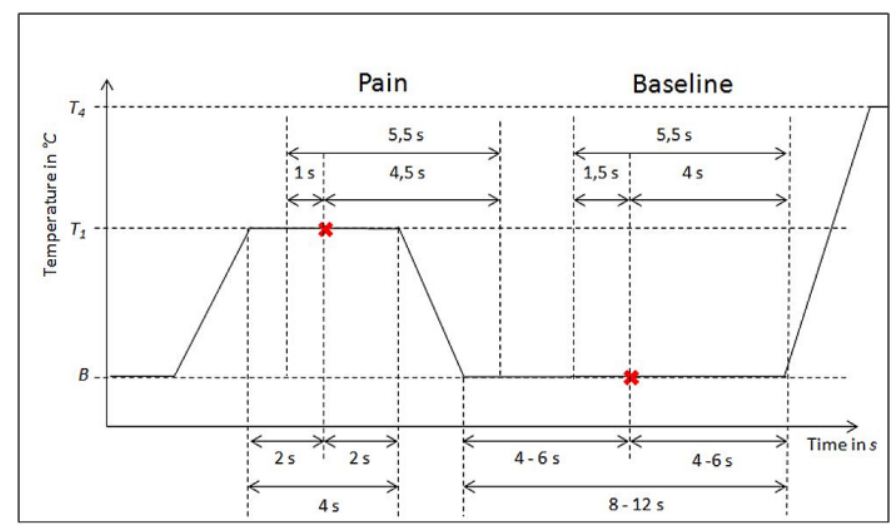

Fig. 1. Computed window for pain quantification [10]

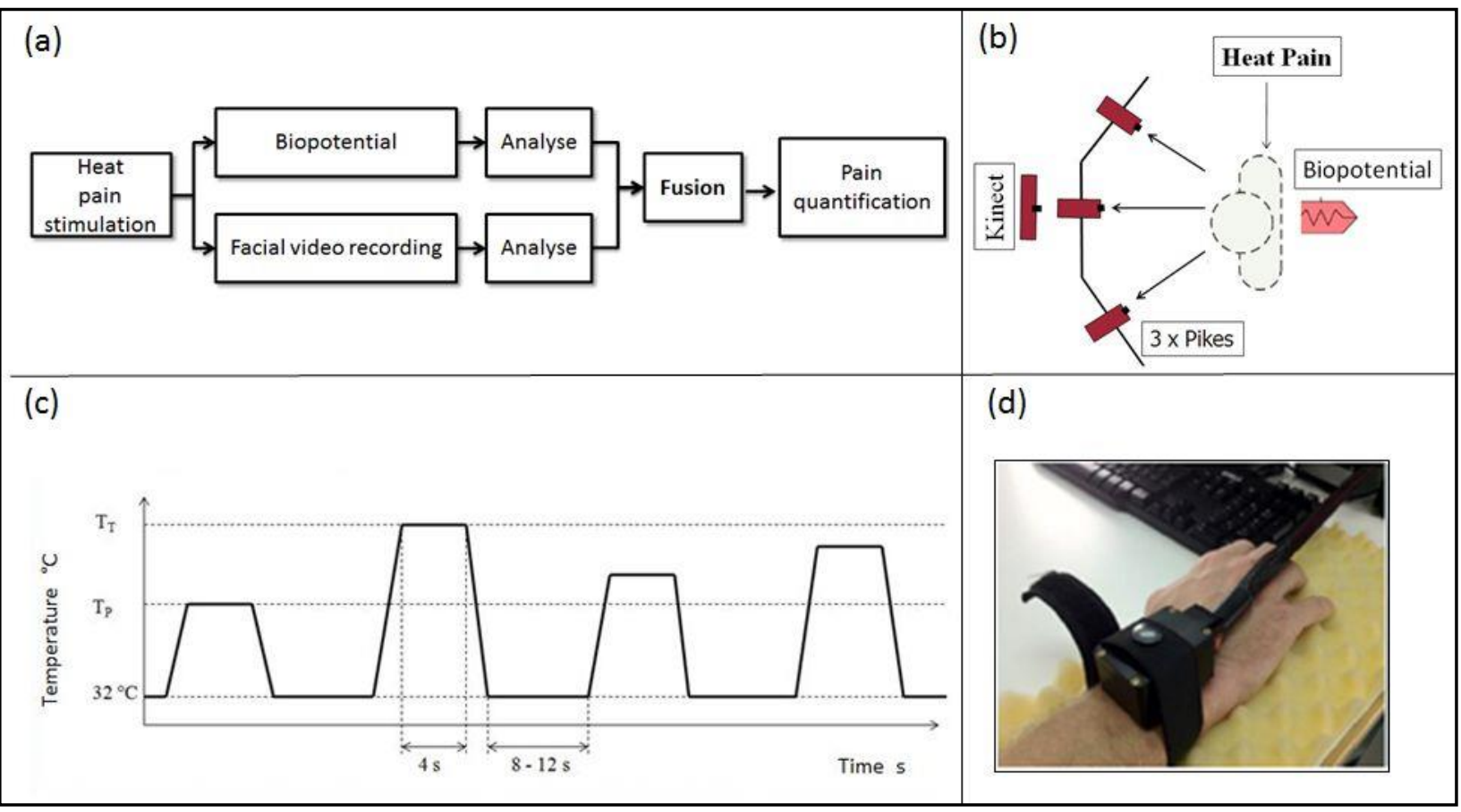

Fig. 2. Experimental design; a. Data processing, b. Lab design, c. Heat stimulation signal, d. Medoc thermode element on the right arm 


\section{CLASSIFICATION AND FUSION APPROACHES}

For the classification of the pain intensity levels, classification and fusion (see figure 2) techniques have been applied to the extracted features $[4,6,8,13]$. The focus of this work is set on the applicability of early and decision fusion approaches for person-independent recognition of pain stimuli. Additionally the combination of early and decision fusion has been investigated by combining specific channels on feature level with level with subsequent fusion with other channels on decision level. Early fusion has been carried out by concatenation of the input feature vectors and subsequent training. Fusion on the decision level relies on decision scores of separate classifiers for each of the channels. In the literature (e.g. [9]) a variety of possible fusion mappings exist (grouped into fixed and trainable). As a fixed mapping we chose the sum rule and as a trainable mapping we decided on a pseudoinverse trained on each of the classifiers' probability outputs per class. The classifier was a Random Forest. Since the dimensionality of the input data (especially when concatenated) reached several hundred, a feature selection algorithm has also been applied - e.g. sequential floating forward selection (SFFS) [11] - to reduce the set to the most discriminative ones.

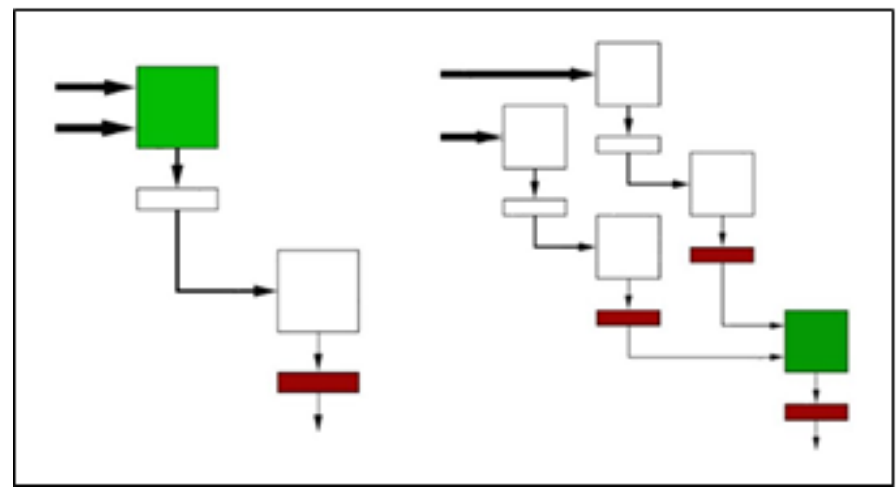

Fig.2. Experimental design; a. early fusion, b. late fusion [8]

\section{RESUlts}

In figure 3 the two class problems of the pain threshold are presented. We made significance test for the comparison of the highest separate parameter with the highest fusion parameter. For the two class problems 0 vs. 4 and 1 vs. 4 , the test of the biopotential (highest separate parameter) vs. late fusion (highest fusion parameter) was significant, for the class two problems 3 vs. 4 the video parameter (highest separate parameter) vs. early fusion, video parameter vs. late fusion was significant.

\section{A. Feature Selection/ Feature Importance}

The biopotential features of facial electromyography and video signals inter_decile_range_of_brow_to_mouth_distance and standard_deviation_of_nasal_wrinkling were chosen as the most selective.

\section{CONCLUSSION}

The simultaneously measurement of both parameters (biopotentials and video) improve the accuracy of pain intensity recognition.

The comparison between the accuracy of biopotential vs. video is not significant. It can be concluded, with the use of only one parameter, both methods have the same effect in relation to the accuracy. Both methods have their pros and cons. In this case, the modality to be omitted could thus be determined by the clinical staff, the patient, or the patient's relatives. The biopotentials are cheaper and well established in clinical institutions. On the negative side, the electrodes which may feel uncomfortable and cosmetically displeasing to the user, must be reattached hourly or daily. Especially for young children could electrodes on the skin elicit anger and anxiety. In contrast, the video method is more comfortable, while the technology is currently expensive and requires ideal lighting conditions.

\section{Outlook}

Further, the classification algorithm requires testing and optimization within a clinical environment. Finally, the goal of the project is the advancement of pain diagnosis and monitoring of pain states. With the use of multimodal sensor technology and highly effective data classification systems, reliable and valid automated pain recognition will be possible. The surrogate measurement of pain with machine learning algorithms will provide valuable information with high temporal resolution for a clinical team, which may help to objectively assess the evolution of treatments (e.g., effect of drugs for pain reduction, information of surgical indication, the quality of care provided to patients).

\section{Acknowledgment}

This research was part of the DFG/TR233/12 "Advancement and Systematic Validation of an Automated Pain Recognition System on the Basis of Facial Expression and Psychobiological Parameters" project, funded by the German Research Foundation, FAPEMIG, CNDq, CAPES, and the Brazilian government. 


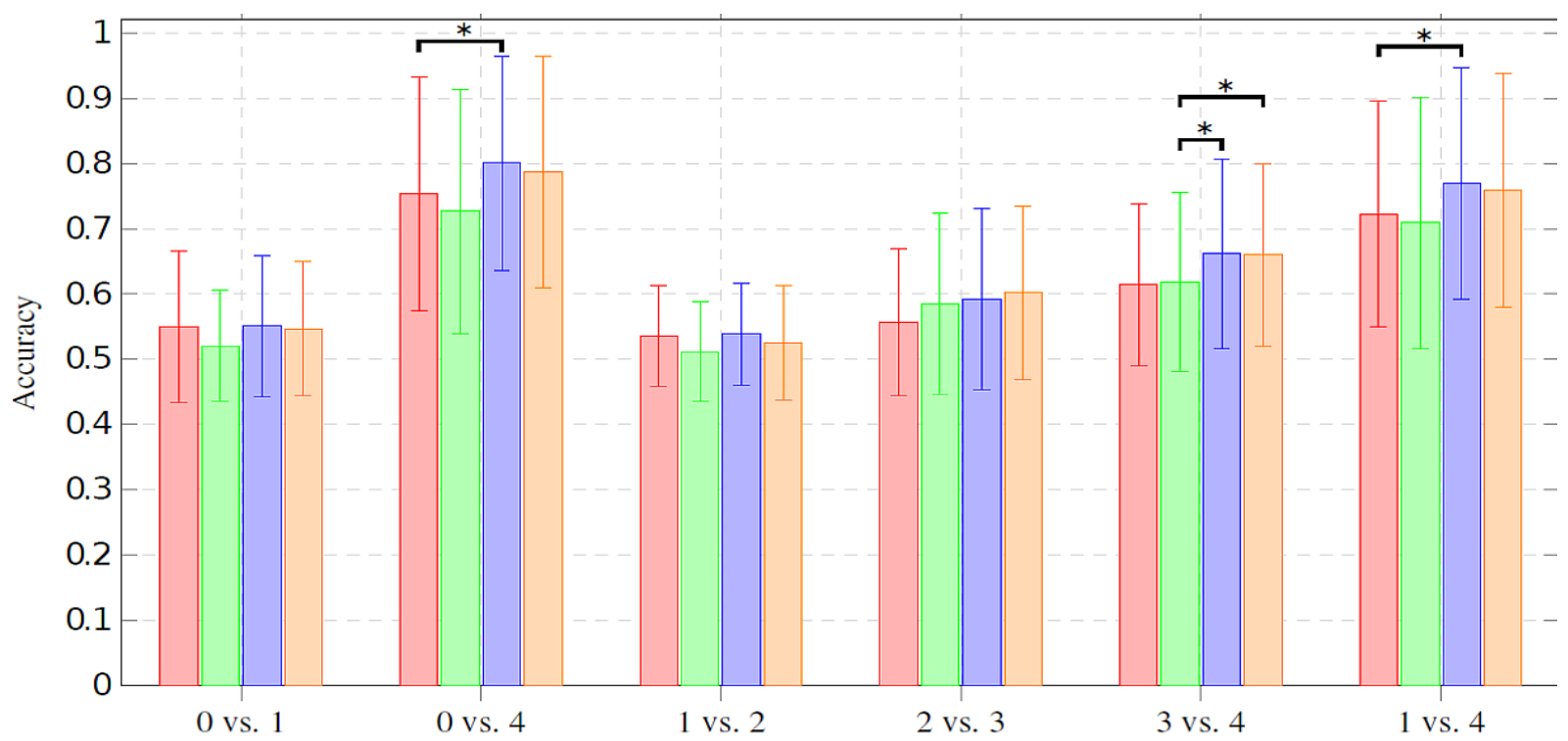

$\square$ Biopotentials $\square$ Video $\square$ Late Fusion $\square$ Early Fusion

Fig. 3. Fusion results via two class problems

[1] A.O. Andrade, P. Kyberd, and S.J. Nasuto, The application of the hilbert spectrum to the analysis of electromyographic signals. Information Sciences 178(9), 2176-2193, 2008.

[2] S. Brahnam, C. Chuang , F. Shih, and M. Slack (2006). "SVM classification of neonatal facial images of pain." Fuzzy Logic and Applications 3849: 121-128.

[3] C. Cao, and S. Slobounov, Application of a novel measure of EEG nonstationarity as 'Shannonentropy of the peak frequency shifting' for detecting residual abnormalities in concussed individuals. Clinical neurophysiology: official journal of the International Federation of Clinical Neurophysiology 122(7), 1314-1321, July 2011.

[4] X.L. Dong, L. Berti-Equille, and D. Srivatava, Data Fusion: Resolving Conflicts from Multiple Sources. In Quality. S. Sadiq (Ed.) Handbook of Data, Berlin Heidelberg: Springer, pp. 293-318, 2013.

[5] C.A. Gilbert, C.M. Lilley, K.D. Craig, P.J. McGrath, C.A. Court, S.M. Bennett, and C.J. Montgomery. Postoperative pain expression in preschool children: Validation of the child facial coding system. Clinical Journal of Pain 15(3): 192-200, 1999.

[6] M. Glodek, S. Reuter, M. Schels, K. Dietmayer, and F. Schwenker. Kalman Filter Based Classifier Fusion for Affective State Recognition. In Z.-H. Zhou, F. Roli and J. Kittler (Eds.) Multiple Classifier Systems., Springer, LNCS 7872, pp. 85-94, 2013.

[7] Z. Hammal, and J.F. Cohn, Automatic detection of pain intensity. In: Proceedings of the $14^{\text {th }}$ ACM International Conference on Multimodal Interaction. pp. 47-52. ICMI '12, ACM, New York, NY, USA, 2012.

[8] M. Kächele, and F. Schwenker, Cascaded fusion of dynamic, spatial, and textural feature sets for person-independent facial emotion recognition. In: Proceedings of the International Conference on Pattern Recognition (ICPR), pp. 4660-4665, 2014.

[9] L. Kuncheva, Combining pattern classifiers: Methods and Algorithms. Wiley, 2004.

[10] P. Lucey, J.F. Cohn, K.M. Prkachin, P.E. Solomon, and I. Matthews, Painful data: The unbc-mcmaster shoulder pain expression archive database. Image, Vision, and Computing Journal, pp. 197-205, 2012.

[11] P. Pudil, J. Novovi cová, and J. Kittler, Floating search methods in feature selection. Pattern Recognition Letters 15(11), 1119-1125, 1994.

[12] J.S. Richman, and J.R. Moorman, Physiological time-series analysis using approximate entropy and sample entropy. Cardiovascular Research 278(6), 2039-2049, 2000.

[13] M. Schels, M. Glodek, S. Meudt, S. Scherer, M. Schmidt, G. Layher, S Tschechne, T. Brosch, D. Hrabal, S. Walter, H.C. Traue, G. Palm, and F. Schwenker, "Multi-Modal Classifier-Fusion for the Recognition of Emotions," in Coverbal Synchrony in Human-Machine Interaction, M. Rojc and N. Campbell, 1 st ed., CRC Press, 2013, pp. 73-98.

[14] R. Treister, M. Kliger, G. Zuckerman, I.G. Aryeh, and E. Eisenberg, Differentiating between heat pain intensities: the combined effect of multiple autonomic parameters. Pain 153(9), 1807-1814, 2012.

[15] S. Walter, S. Gruss, K. Limbrecht, H.C. Traue, P. Werner, A. AlHamadi, N. Diniz, G. Moreira, and A.O. Andrade, Automatic pain quantification using autonomic parameters. Psychology \& Neuroscience 7(3): 363-380, 2014.

[16] S. Walter, S. Gruss, H. Ehleiter, J. Tan, H. Traue, P. Werner, A. AlHamadi, S. Crawcour, A. Andrade, and G. Moreira da Silva, The BioVid heat pain database data for the advancement and systematic validation of an automated pain recognition system. In: Cybernetics (CYBCONF), 2013 IEEE International Conference on. pp. 128-131, June 2013.

[17] P. Werner, A. Al-Hamadi, R. Niese, S. Walter, S. Gruss, and H.C. Traue, Towards pain monitoring: Facial expression, head pose, a new database, an automatic system and remaining challenges. In: Proceedings of the British Machine Vision Conference. pp. 119.1119.13. BMVA Press, 2013.

[18] P. Werner, A. Al-Hamadi, R. Niese, S. Walter, S. Gruss, and H.C. Traue, Automatic pain recognition from video and biomedical signals. In: International Conference on Pattern Recognition. pp. 4582-4587, 2014.

[19] X. Xiong, and F. De la Torre, Supervised descent method and its applications to face alignment. In: Computer Vision and Pattern Recognition (CVPR), 2013 IEEE Conference on. pp. 532-539, 2013.

[20] S.M. Zwakhalen, J.P. Hamers, H.H. Abu-Saad, and M.P. Berger, Pain in elderly people with severe dementia: a systematic review of behavioral pain assessment tools. BMC Geriatr 6: 3, 2006. 